\title{
Survival impact of time to initiation of chemoradiotherapy after resection of newly diagnosed glioblastoma
}

\author{
Matthew Z. Sun, BS, ${ }^{1}$ Taemin Oh, BA, ${ }^{2}$ Michael E. Ivan, MD, MBS, ${ }^{1}$ Aaron J. Clark, MD, PhD, ${ }^{1}$ \\ Michael Safaee, MD, ${ }^{1}$ Eli T. Sayegh, BS, ${ }^{2}$ Gurvinder Kaur, MD, ${ }^{2}$ Andrew T. Parsa, MD, PhD, ${ }^{2}$ and \\ Orin Bloch, MD²

\begin{abstract}
1Department of Neurological Surgery, University of California, San Francisco, California; and 'Department of Neurological Surgery, Northwestern University Feinberg School of Medicine, Chicago, Illinois
\end{abstract}

\begin{abstract}
OBJECT There are few and conflicting reports on the effects of delayed initiation of chemoradiotherapy on the survival of patients with glioblastoma. The standard of care for newly diagnosed glioblastoma is concurrent radiotherapy and temozolomide chemotherapy after maximal safe resection; however, the optimal timing of such therapy is poorly defined. Given the lack of consensus in the literature, the authors performed a retrospective analysis of The Cancer Genome Atlas (TCGA) database to investigate the effect of time from surgery to initiation of therapy on survival in newly diagnosed glioblastoma.
\end{abstract}

METHODS Patients with primary glioblastoma diagnosed since 2005 and treated according to the standard of care were identified from TCGA database. Kaplan-Meier and multivariate Cox regression analyses were used to compare overall survival (OS) and progression-free survival (PFS) between groups stratified by postoperative delay to initiation of radiation treatment.

RESULTS There were 218 patients with newly diagnosed glioblastoma with known time to initiation of radiotherapy identified in the database. The median duration until therapy was 27 days. Delay to radiotherapy longer than the median was not associated with worse PFS ( $H R=0.918, p=0.680)$ or $O S(H R=1.135, p=0.595)$ in multivariate analysis when controlling for age, sex, KPS score, and adjuvant chemotherapy. Patients in the highest and lowest quartiles for delay to therapy ( $\leq 20$ days vs $\geq 36$ days) did not statistically differ in PFS $(p=0.667)$ or $O S(p=0.124)$. The small subset of $p a-$ tients with particularly long delays (> 42 days) demonstrated worse OS $(H R=1.835, p=0.019)$, but not PFS $(p=0.74)$.

CONCLUSIONS Modest delay in initiation of postoperative chemotherapy and radiation does not appear to be associated with worse PFS or OS in patients with newly diagnosed glioblastoma, while significant delay longer than 6 weeks may be associated with worse OS.

http://thejns.org/doi/abs/10.3171/2014.9.JNS14193

KEY WORDS glioblastoma; overall survival; progression-free survival; treatment delay; The Cancer Genome Atlas; oncology

$\mathrm{R}$ ANDOMIZED clinical trials have established the efficacy of radiation and temozolomide chemotherapy for newly diagnosed glioblastoma. ${ }^{14,15}$ The standard of care for newly diagnosed glioblastoma consists of maximum safe resection of the tumor followed by radiotherapy and concurrent temozolomide based on the results of a randomized Phase III trial reported by Stupp and colleagues in $2005 .{ }^{15}$ However, there is a paucity of data on the appropriate timing of chemoradiotherapy after surgery. The Stupp trial included patients who initiated therapy ranging from 1.7 to 10.7 weeks after diagnosis, with a median delay of 5 weeks. ${ }^{15}$

Given the aggressive nature of glioblastoma, radiotherapy and temozolomide are often recommended to start at the shortest safe interval following resection. A window of 2-3 weeks following surgery is considered ideal, al-

ABBREVIATIONS $\mathrm{CI}=$ confidence interval; $\mathrm{HR}=$ hazard ratio; KPS = Karnofsky Performance Scale; OS = overall survival; PFS = progression-free survival; TCGA = The Cancer Genome Atlas.

SUBMITTED January 24, 2014. ACCEPTED September 18, 2014.

INCLUDE WHEN CITING Published online March 13, 2015; DOI: 10.3171/2014.9.JNS14193.

DISCLOSURE M. Z. Sun is a Howard Hughes Medical Institute Medical Research Fellow. Dr. Safaee was supported by a grant from the Doris Duke Charitable Foundation. Dr. Ivan is funded by the National Research Education Foundation through the AANS. Dr. Parsa is the Michael J. Marchese endowed chair of Neurological Surgery. Dr. Bloch is the Khatib Professor of Neurological Surgery. 
lowing sufficient time for postoperative recovery without excessively delaying therapy. While there is no definitive evidence supporting this recommendation, mathematical modeling of the growth of residual tumor after surgery estimates a doubling time of 24 days without chemoradiotherapy. ${ }^{2}$ Thus, there is a reasonable concern for tumor progression or recurrence before commencement of therapy.

Despite the concerns regarding the delay until initiation of chemoradiotherapy for glioblastoma, there is little conclusive data available. Most of the published studies evaluated patients treated prior to the modern era of concurrent temozolomide and radiotherapy, and showed that delay of radiation had varying effects on survival., ${ }^{1,4,9,10,16}$ There are only 2 studies that analyzed patients treated exclusively during the modern era, but they showed different findings. Noel et al. found that in a cohort of 400 patients in France there was no effect of delay to therapy on survival, ${ }^{12}$ while Graus et al. found in 396 patients in Spain that $\leq 42$ days of delay was associated with better progression-free survival (PFS) but not overall survival (OS). ${ }^{6}$

To help clarify this issue, we conducted a retrospective analysis of a modern cohort of patients treated under the current standard of care. Patient data collected in The Cancer Genome Atlas (TCGA) database ${ }^{3}$ was used to assess the impact of delay to therapy on survival for patients with newly diagnosed glioblastoma.

\section{Methods \\ Patient Selection}

Patients with newly diagnosed glioblastoma treated during the modern era were identified from TCGA database. Patients treated before 2005 or without concurrent radiation and temozolomide chemotherapy were excluded. The remaining patients undergoing resection and standard chemoradiotherapy with data available on the time to radiation and OS were included in the study.

\section{Data Extraction}

For each patient the age, sex, preoperative Karnofsky Performance Scale (KPS) score, length of delay from surgery to radiation, OS, and PFS were recorded. The use of additional primary or adjuvant chemotherapy was also recorded.

\section{Statistical Analysis}

The effect of each demographic factor on PFS and OS was analyzed by the Kaplan-Meier method with differences assessed by a log-rank test. A multivariate Cox proportional hazards model was fitted by stepwise enter forward selection while accounting for potentially confounding variables, including sex, age, preoperative KPS score, and the use of additional chemotherapy. Age was treated as a continuous variable, while sex and use of additional chemotherapy were treated as nominal variables. KPS score was defined as a dichotomous variable, with patients divided into groups of KPS score $\leq 70$ or KPS score $>70$. Means of continuous variables were analyzed using ANOVA, and categorical values were analyzed using the Pearson chi-square test. The Fisher exact test was used if the expected cell count in a contingency table was less than 5 . All $\mathrm{p}$ values $<0.05$ were considered statistically significant. These analyses were performed using the statistical software package SPSS (version 20, IBM Inc.).

A post hoc power analysis was performed to evaluate the results, utilizing a minimum power of 0.8 and $\alpha$ of 0.05 to determine the necessary sample size to define a significant difference in outcomes.

\section{Results \\ Patient Demographics}

There were 218 patients with newly diagnosed glioblastoma treated with standard of care chemotherapy and radiation for whom time to initiation of therapy was available. This cohort included 140 men and 78 women, with a median age of 58 years (range 21-86 years). The median KPS score was 80 (range 20-100). Radiotherapy was delivered to all patients at a median dose of $60 \mathrm{~Gy}$. All patients received concurrent temozolomide chemotherapy, and additional chemotherapy was given at tumor recurrence to 74 patients. The mean follow-up duration was 420 days.

Delay to therapy was defined as the time from surgery to the initiation of radiotherapy. The median delay was 27 days, with a range of 7 to 232 days (Fig. 1). For statistical analysis, patients were stratified by length of delay to therapy relative to the median time to therapy. Additionally, data were analyzed by grouping patients into quartiles of delay to therapy, with the first quartile including all patients with delays up to 20 days, the second quartile including 21-27 days, the third quartile including 28-35 days, and the fourth quartile including 36 days or longer. Patient demographics were compared between the stratification groups. Patients with a delay to therapy longer or shorter than the median demonstrated no differences in age, male to female ratio, KPS score, or use of additional chemotherapy at tumor recurrence (Table 1). Comparing

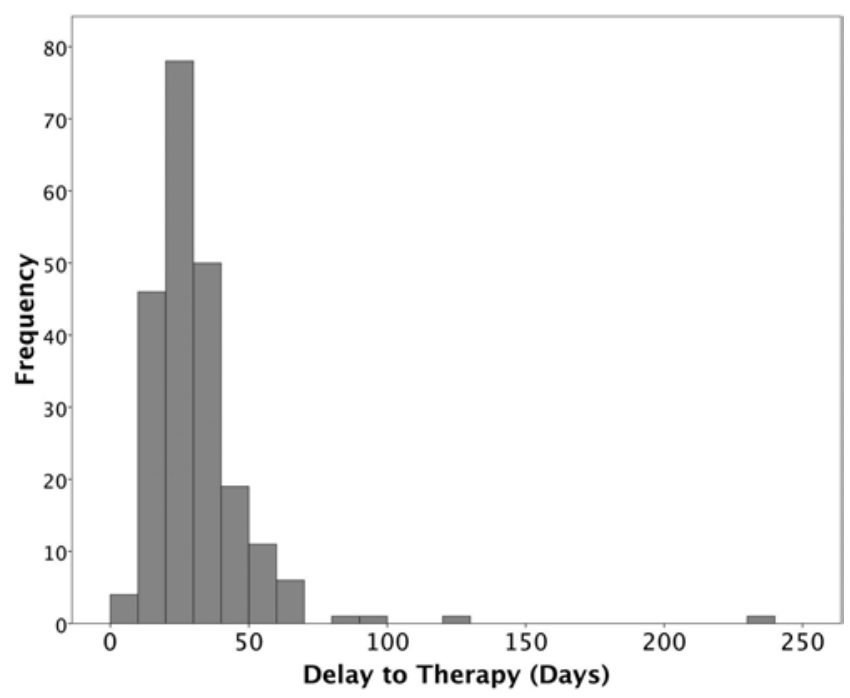

FIG. 1. Graph of the distribution of delay of adjuvant therapy. The distribution of 218 patients with newly diagnosed glioblastoma from TCGA with a known length of delay was plotted in a histogram. Each interval represents 10 days of delay, which is defined as the number of days to initiating radiation after surgery. 
TABLE 1. Patient demographics according to delay to treatment*

\begin{tabular}{|c|c|c|c|}
\hline \multirow[b]{2}{*}{ Category } & \multicolumn{2}{|c|}{ Treatment Delay } & \multirow[b]{2}{*}{ p Value } \\
\hline & $\leq 27$ Days & $>27$ Days & \\
\hline Total $(n=218)$ & 113 & 105 & \\
\hline Mean age & 57.2 & 58.6 & 0.437 \\
\hline \multicolumn{4}{|l|}{ Sex } \\
\hline Female & 34 & 44 & 0.090 \\
\hline Male & 79 & 61 & \\
\hline \multicolumn{4}{|l|}{ KPS Score } \\
\hline$\leq 70$ & 18 & 19 & 0.578 \\
\hline$>70$ & 75 & 61 & \\
\hline \multicolumn{4}{|c|}{ Chemotherapy after recurrence } \\
\hline No & 60 & 67 & 0.307 \\
\hline Yes & 41 & 33 & \\
\hline \multicolumn{4}{|l|}{ Gliadel } \\
\hline No & 106 & 93 & 0.314 \\
\hline Yes & 3 & 6 & \\
\hline \multicolumn{4}{|l|}{ Bevacizumab } \\
\hline No & 68 & 74 & 0.073 \\
\hline Yes & 41 & 25 & \\
\hline \multicolumn{4}{|l|}{ Lomustine } \\
\hline No & 100 & 93 & 0.600 \\
\hline Yes & 9 & 6 & \\
\hline \multicolumn{4}{|l|}{ Carmustine } \\
\hline No & 108 & 96 & 0.349 \\
\hline Yes & 1 & 3 & \\
\hline \multicolumn{4}{|l|}{ Other adjuvants } \\
\hline No & 65 & 71 & 0.080 \\
\hline Yes & 44 & 28 & \\
\hline
\end{tabular}

* Age, sex, postoperative KPS score, use of additional chemotherapy after tumor recurrence, and use of Gliadel, bevacizumab, lomustine, carmustine, and other adjuvant therapy were compared between those with shorter and longer than median delay ( $\leq 27$ days vs $>27$ days, respectively).

the same parameters between patients stratified by quartile of delay to therapy, there were also no significant differences between patients in the first and fourth quartiles (Table 2).

\section{Progression-Free Survival}

The impact of delay to initiation of therapy on PFS was assessed univariately by comparing PFS in patients with delays shorter than or equal to the median $(\leq 27$ days) to those patients with delays greater than the median (> 27 days) by Kaplan-Meier analysis (Fig. 2 left). Median PFS was 7.2 months $(95 \%$ confidence interval [CI] 5.5-8.9 months) in patients with a delay shorter than the median, as compared with 7.8 months (95\% CI 5.5-10.0 months) in patients with a delay longer than the median. The difference between the groups was not significant $(\mathrm{p}$ $=0.840$ ). To evaluate the extremes of delayed therapy, PFS in patients in the shortest and longest quartiles of delay to therapy were compared (Fig. 2 right). Again, differences between the 2 groups were not significant, with a median PFS of 5.9 months (95\% CI 4.6-7.1 months) in the short-
TABLE 2. Patient demographics according to quartile of delay to therapy*

\begin{tabular}{|c|c|c|c|}
\hline Category & $\begin{array}{l}\text { 1st Quartile } \\
\text { ( } \leq 20 \text { days) }\end{array}$ & $\begin{array}{l}\text { 4th Quartile } \\
\text { ( } \geq 36 \text { days) }\end{array}$ & $p$ Value \\
\hline Total $(n=108)$ & 56 & 52 & \\
\hline Mean age & 58.6 & 59.0 & 0.866 \\
\hline \multicolumn{4}{|l|}{ Sex } \\
\hline Female & 16 & 18 & 0.539 \\
\hline Male & 40 & 34 & \\
\hline \multicolumn{4}{|l|}{ KPS score } \\
\hline$\leq 70$ & 12 & 11 & 1.000 \\
\hline$>70$ & 34 & 35 & \\
\hline \multicolumn{4}{|c|}{ Chemotherapy after recurrence } \\
\hline No & 30 & 31 & 1.000 \\
\hline Yes & 18 & 17 & \\
\hline \multicolumn{4}{|l|}{ Gliadel } \\
\hline No & 53 & 49 & 0.611 \\
\hline Yes & 1 & 2 & \\
\hline \multicolumn{4}{|l|}{ Bevacizumab } \\
\hline No & 36 & 38 & 0.401 \\
\hline Yes & 18 & 13 & \\
\hline \multicolumn{4}{|l|}{ Lomustine } \\
\hline No & 50 & 50 & 0.364 \\
\hline Yes & 4 & 1 & \\
\hline \multicolumn{4}{|l|}{ Carmustine } \\
\hline No & 54 & 49 & 0.234 \\
\hline Yes & 0 & 2 & \\
\hline \multicolumn{4}{|l|}{ Other adjuvants } \\
\hline No & 32 & 37 & 0.217 \\
\hline Yes & 22 & 14 & \\
\hline
\end{tabular}

* Age, sex, postoperative KPS score, use of additional chemotherapy after tumor recurrence, and use of Gliadel, bevacizumab, lomustine, carmustine, and other adjuvant therapy were compared between those with first and fourth quartile of delay ( $\leq 20$ days vs $\geq 36$ days, respectively).

est quartile ( $\leq 20$ days) compared with 7.4 months (95\% CI 4.3-10.9 months) in the longest quartile ( $\geq 36$ days) of delay $(\mathrm{p}=0.667)$.

A multivariate Cox proportional hazards model was developed to evaluate the impact of delay to therapy while controlling for age, sex, KPS score, and use of additional chemotherapy at tumor recurrence (Table 3). Comparing groups with a delay to therapy greater than or less than the median, delay time was not found to be predictive of PFS, with a hazard ratio (HR) of 0.918 (95\% CI 0.611-1.379, p $=0.680$ ). Only KPS score was found to be an independent predictor of outcome with an HR of 0.564 (95\% CI $0.333-0.956, p=0.033$ ) for preoperative KPS score $>70$ compared with $\leq 70$.

A post hoc power analysis was performed to evaluate the power of the data to evaluate differences in PFS. The baseline PFS of patients with a short delay to therapy was 7.2 months. A difference in PFS of 4 or more weeks among patients with a longer delay was defined as clinically significant. Under these assumptions, a sample size 

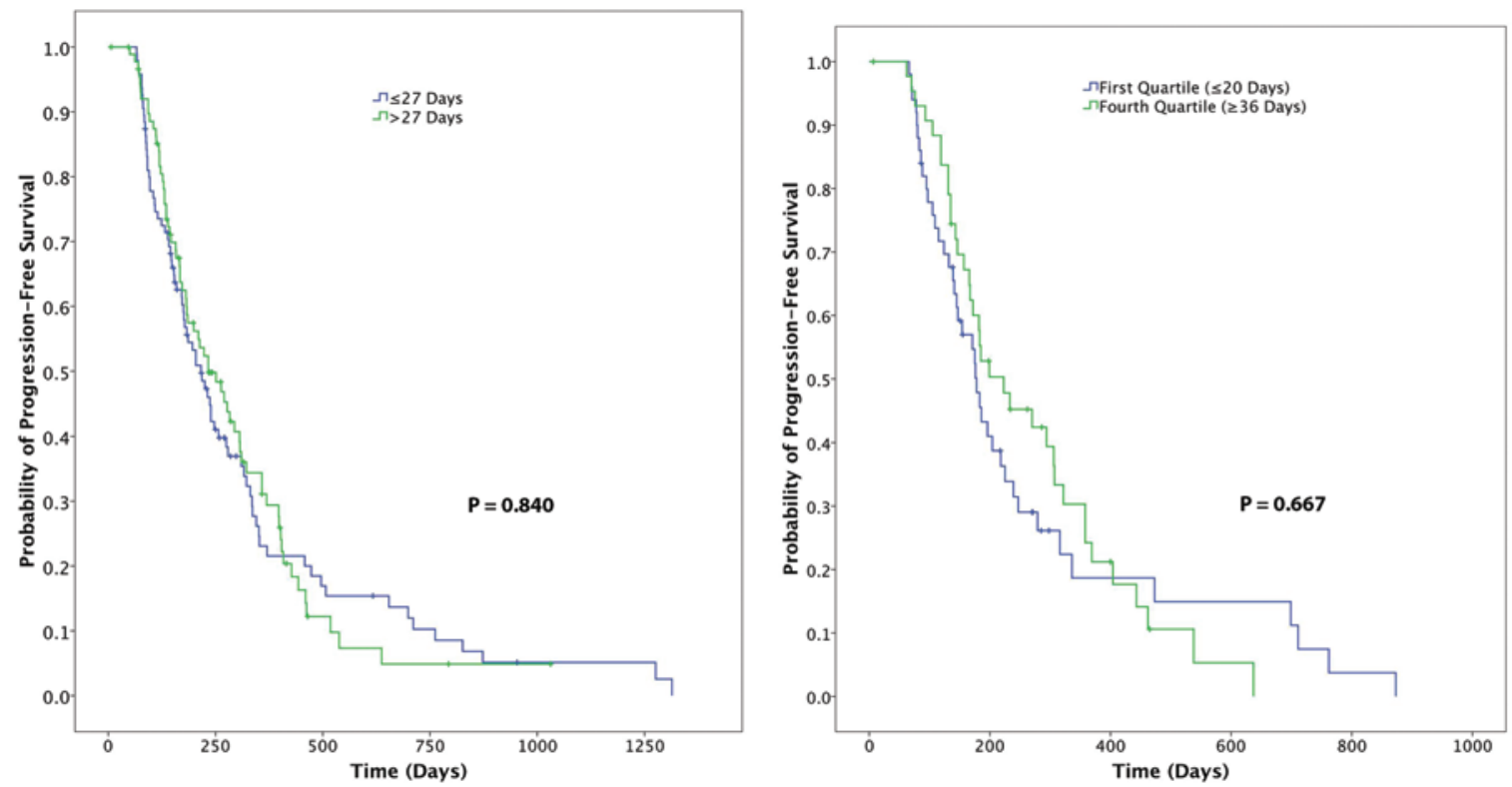

FIG. 2. Graphs showing that delay of adjuvant therapy is not associated with worse PFS. Left: Kaplan-Meier analysis of PFS between those with shorter than median delay ( $\leq 27$ days) and those with longer than median delay ( $>27$ days). Right: KaplanMeier analysis of PFS between those in the first quartile ( $\leq 20$ days) and fourth quartile ( $\geq 36$ days) of delay. Figure is available in color online only.

of 216 patients would have a power of $80 \%$ to identify a clinically significant difference in PFS.

Although the intended analysis of PFS was to compare patients relative to the median delay in therapy, previous studies have suggested that significant delays of greater than 42 days (6 weeks) were associated with decreased survival. ${ }^{16}$ Therefore, PFS was also compared between patients with $>42$ days delay to therapy and patients with $\leq$ 42 days delay. There were no observed differences in PFS between groups when evaluated univariately $(\mathrm{p}=0.61$, log-rank test; Fig. 3 right) or multivariately ( $=0.74)$.

\section{Overall Survival}

The impact on OS of delay to initiation of therapy was

TABLE 3. Impact of modest treatment delay on PFS*

\begin{tabular}{llc}
\hline \multicolumn{1}{c}{ Variable } & HR $(95 \% \mathrm{Cl})$ & $\mathrm{p}$ Value \\
\hline Delay & $0.918(0.611-1.379)$ & 0.680 \\
\hline Age & $1.008(0.992-1.023)$ & 0.334 \\
\hline Sex & $1.017(0.651-1.590)$ & 0.941 \\
\hline KPS score & $0.564(0.333-0.956)$ & 0.033 \\
\hline Chemotherapy at recurrence & $1.745(0.953-3.195)$ & 0.071 \\
\hline Gliadel & $2.275(0.432-11.978)$ & 0.332 \\
\hline Bevacizumab & $0.883(0.495-1.573)$ & 0.672 \\
\hline Lomustine & $0.970(0.456-2.064)$ & 0.937 \\
\hline Carmustine & $1.376(0.333-5.697)$ & 0.659 \\
\hline Other adjuvant & $0.998(0.617-1.615)$ & 0.993 \\
\hline
\end{tabular}

* Multivariate Cox regression analysis controlling for age, sex, postoperative KPS score, use of additional chemotherapy after tumor recurrence, and use of Gliadel, bevacizumab, lomustine, carmustine, and other adjuvant therapy was performed to compare the effects of delay on PFS between those with shorter and those with longer than median delay ( $\leq 27$ days vs $>27$ days, respectively). assessed univariately by comparing OS in patients with delays shorter than or equal to the median ( $\leq 27$ days) with patients with delays greater than the median $(>27$ days) by Kaplan-Meier analysis (Fig. 4 left). Median OS was 15.9 months (95\% CI 13.5-18.3 months) in patients with a delay shorter than the median, as compared with 14.9 months (95\% CI 14.0-15.9 months) in patients with a delay longer than the median. The difference between the groups was not significant ( $\mathrm{p}=0.180)$. To evaluate the extremes of delayed therapy, OS in patients in the shortest and longest quartiles of delay to therapy were compared (Fig. 4 right). Again, differences between groups were not significant, with a median OS of 16.0 months (95\% CI 8.3-23.7 months) in the shortest quartile ( $\leq 20$ days) compared with 14.2 months (95\% CI 12.1-16.4 months) in the longest quartile ( $\geq 36$ days) of delay $(p=0.124)$.

A multivariate Cox proportional hazards model was developed to evaluate the impact of delay to therapy while controlling for age, sex, KPS score, and use of additional chemotherapy at tumor recurrence (Table 4). Comparing groups with a delay to therapy greater than or less than the median, delay time was not found to be predictive of OS, with an HR of 1.135 (95\% CI 0.711-1.813, p = 0.595). Only age was found to be an independent predictor of outcome, with an HR of 1.018 (95\% CI 1.001-1.036, p = 0.049) per incremental year.

Because previous reports have suggested that greater than 42 days delay to therapy significantly impacts survival, OS was also evaluated in the small subset of patients with $>42$ days delay to therapy and compared with patients with $\leq 42$ days delay. Univariate Kaplan-Meier analysis of OS showed a significant difference of 15.9 versus 12.9 months ( $\mathrm{p}=0.022$; Fig. 3 left). Multivariate Cox regression showed that longer delay had an HR of death of 1.835 (95\% CI 1.104-3.051, $\mathrm{p}=0.019)$. 

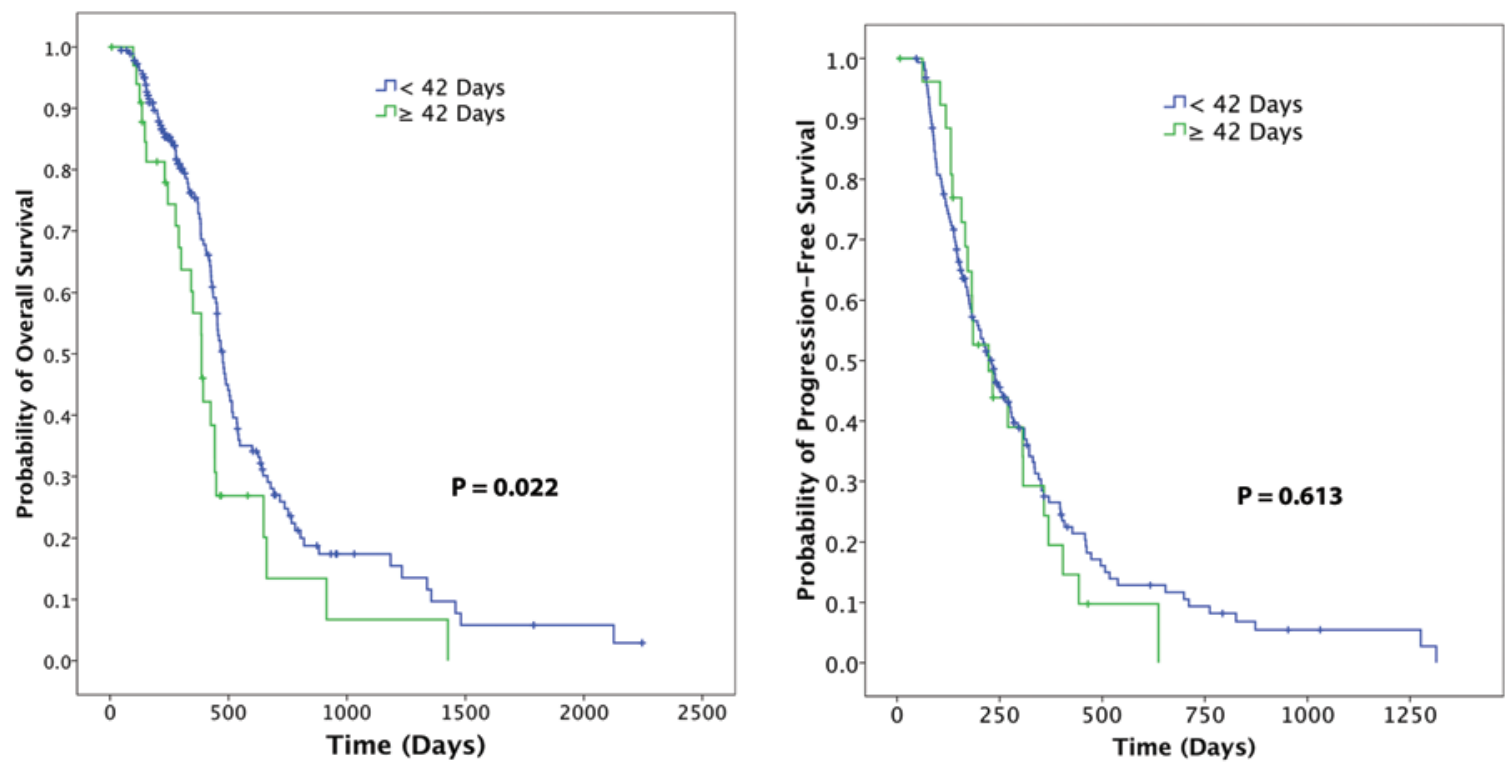

FIG. 3. Graphs showing significant delay of adjuvant therapy is associated with worse OS but not PFS. Left: Kaplan-Meier analysis of OS between those with treatment delay of longer and shorter than 42 days. Right: Kaplan-Meier analysis of PFS between those with treatment delay of longer and shorter than 42 days. Figure is available in color online only.

\section{Discussion}

Long delays between resection and initiation of therapy can cause anxiety in both patients with glioblastoma and in providers who may be concerned about interval tumor progression. ${ }^{8}$ However, the current literature lacks definitive evidence demonstrating that delay in the initiation of therapy has an adverse effect on survival. Moreover, the existing data are derived almost exclusively from European patient populations, largely treated before the modern era of concurrent temozolomide and radiation therapies. While most of these studies showed that delay of radiation therapy had no effect on survival, ${ }^{1,4,9,10}$ a single study by Irwin et al. showed that the risk of death (HR) increased by $8.9 \%$ for every week of delay to initiation of radiotherapy. ${ }^{7}$ In contrast, however, Blumenthal et al. not only found that delay was not associated with worse survival, but that the longest delayed group had the best OS.' Only a few studies have been published that include patients treated after 2005 with the current standard of care. Valduvieco et al. found that a greater than 6-week delay was associated with worse OS in a Spanish population. ${ }^{16}$ However, this study also included some patients who were treated before
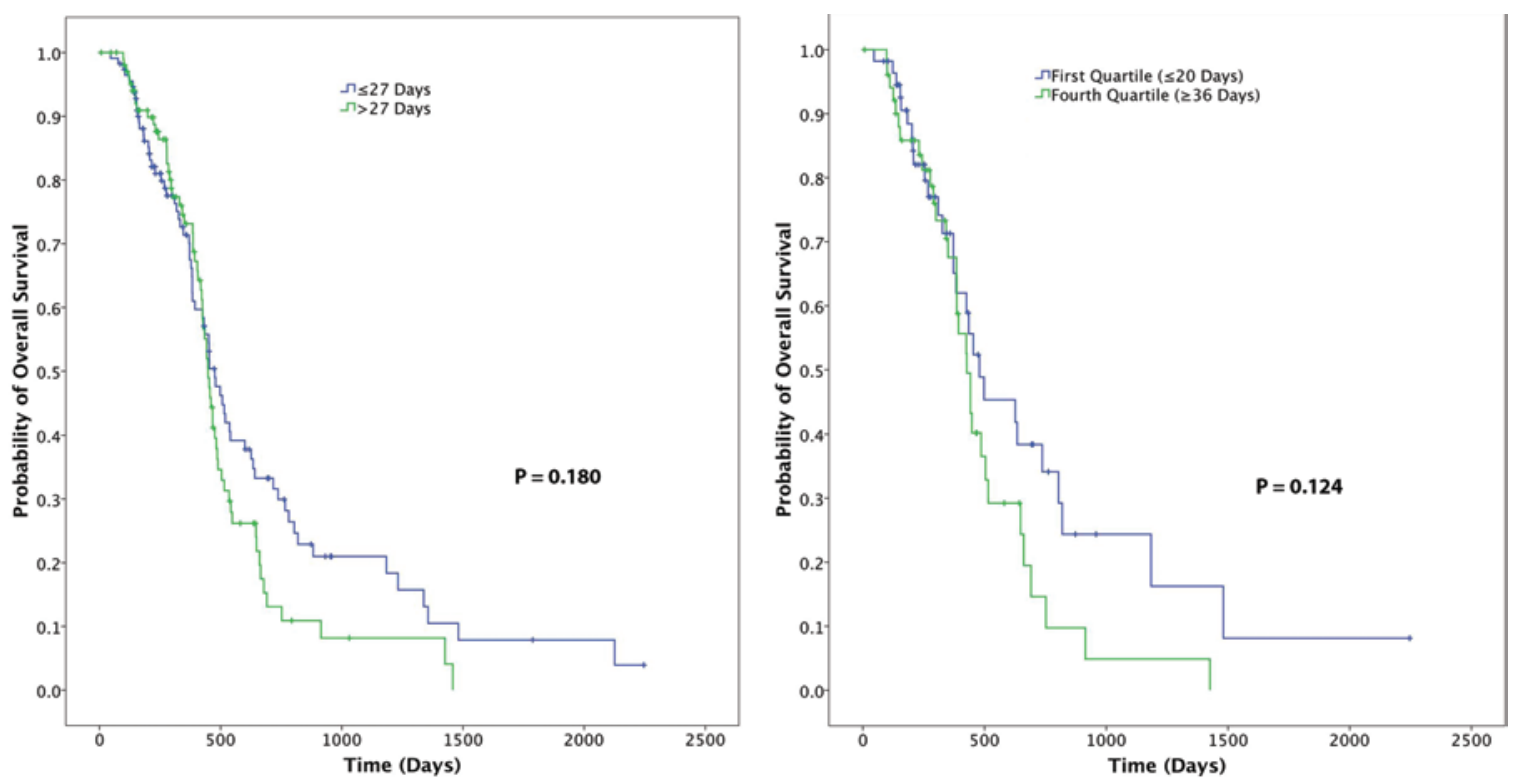

FIG. 4. Graphs showing modest delay of adjuvant therapy is not associated with worse OS. Left: Kaplan-Meier analysis of OS between those with shorter than median delay ( $\leq 27$ days) and those with longer than median delay (> 27 days). Right: KaplanMeier analysis of OS between those in the first quartile ( $\leq 20$ days) and fourth quartile ( $\geq 36$ days) of delay. Figure is available in color online only. 
TABLE 4. Impact of modest treatment delay on OS*

\begin{tabular}{lcc}
\hline \multicolumn{1}{c}{ Variable } & $\mathrm{HR}(95 \% \mathrm{Cl})$ & $\mathrm{p} \mathrm{Value}$ \\
\hline Delay & $1.135(0.711-1.813)$ & 0.595 \\
\hline Age & $1.018(1.001-1.036)$ & 0.049 \\
\hline Sex & $1.344(0.788-2.292)$ & 0.279 \\
\hline KPS score & $0.599(0.321-1.118)$ & 0.108 \\
\hline Chemotherapy at recurrence & $0.925(0.515-1.661)$ & 0.794 \\
\hline Gliadel & $1.842(0.484-7.018)$ & 0.370 \\
\hline Bevacizumab & $0.741(0.407-1.349)$ & 0.327 \\
\hline Lomustine & $1.876(0.906-3.888)$ & 0.090 \\
\hline Carmustine & $0.719(0.155-3.333)$ & 0.673 \\
\hline Other adjuvant & $0.763(0.454-1.281)$ & 0.306 \\
\hline
\end{tabular}

* Multivariate Cox regression analysis controlling for age, sex, postoperative KPS score, use of additional chemotherapy after tumor recurrence, Gliadel, bevacizumab, lomustine, carmustine, and other adjuvant therapy was performed to compare the effects of delay on OS between those with shorter and those with longer than median delay ( $\leq 27$ days vs $>27$ days, respectively).

the modern era, as patients were diagnosed between 1994 and 2009. ${ }^{16}$ Noel et al. found in a cohort of 400 French patients, all of whom were treated during the modern protocol era, that there was no effect on survival associated with delay. ${ }^{11}$ More recently, Graus et al. found that in a cohort of 396 Spanish patients, initiation of radiotherapy within 42 days of surgery was associated with longer PFS but not with OS. ${ }^{6}$ It appears there is a lack of consensus on the effects of delay to initial therapy from studies of patients treated both before and after the modern protocol era, and no studies on patients treated during the Stupp protocol era have been conducted outside of Europe.

Using pooled data from TCGA database, ${ }^{3}$ we analyzed the effect of delay to initial therapy on survival in a contemporary group of patients with glioblastoma from North America. This large dataset was made up primarily of patients from large academic centers treating patients with glioblastoma according to the modern standard of care. ${ }^{3,13,17}$ In this population, we found that modest delays to initiation of chemoradiotherapy of 5.2 weeks or more, corresponding to the fourth quartile in TCGA database, were not associated with worse PFS or OS. The lack of impact of delay to therapy on survival remained after analysis relative to the median or in quartiles, and in both univariate and multivariate analysis. As expected, the multivariate models identified KPS score as an independent predictor of PFS and age as an independent predictor of OS, serving as internal validations of the models. However, these models did not demonstrate a statistical association with delay to therapy.

In the small subset of patients with delays longer than 42 days (6 weeks), delay to therapy was associated with worse OS, similar to what was reported by Valduvieco et al. in a Spanish contemporary cohort. ${ }^{16}$ While a delay greater than 42 days impacted OS, there was no significant difference in PFS. Because data regarding the reasons for delay to therapy were not available in the data set, we cannot assess whether the small group of patients with the most extreme delay to therapy suffered from comorbid conditions that could confound their poor survival. Overall, our findings are consistent with the majority of similar studies over the past decade. ${ }^{1,4,9,10}$ An older retrospective study conducted by the Radiation Therapy Oncology Group actually found that patients who underwent radiation treatment more than 4 weeks postoperatively had a statistically significant survival advantage over the group that started $\leq 2$ weeks postoperatively $(\mathrm{HR}=0.84, \mathrm{p}<0.003) .{ }^{1}$ We did not observe this finding, which predates the modern standard of concurrent chemotherapy and radiation, when comparing patients in the shortest and longest quartiles of delay in our population.

There are significant limitations to the current study, largely arising from the retrospective design and limitations that are inherent in the use of a large multiinstitutional database that was not originally created for the purposes of this study. Using data from TCGA database, we were limited with the number of possible confounding variables that could be accounted for in the multivariate model. Specifically, we were not able to control for extent of resection, tumor location, or tumor size. ${ }^{5}$ Additionally, we were not able to control for the extent of initial temozolomide chemotherapy received or additional experimental therapy due to enrollment in clinical trials. Furthermore, information regarding the reason for delay was unavailable. Although the ideal method to address the impact of delay to therapy would be through a prospective randomized trial, it is difficult to justify randomizing patients to increased delay once they are ready to initiate chemotherapy and radiation. Additionally, a well-defined, prospective, multicenter registry including data on delay to therapy, extent of resection, and molecular tumor markers would contribute important insight into the acceptable length of delay to initial therapy. However, until such studies are performed, we must rely on retrospective data from large, validated databases such as TCGA to make treatment recommendations. Given the results of this study and others, there does not appear to be a survival cost to modestly delaying initiation of chemoradiotherapy following resection of newly diagnosed glioblastoma, within a 6-week window. It is, therefore, reasonable to delay therapy long enough to optimize patients' wounds and functional status without a concern about impact on outcomes.

\section{Conclusions}

Modest delays of up to 6 weeks in the initiation of chemotherapy and radiation following resection of newly diagnosed glioblastoma are not associated with worsened PFS or OS. However, significant delays longer than 6 weeks may negatively affect OS. Therapy can safely be delayed up to 6 weeks to optimize patients' status or address social concerns without negatively affecting outcome.

\section{References}

1. Blumenthal DT, Won M, Mehta MP, Curran WJ, Souhami L, Michalski JM, et al: Short delay in initiation of radiotherapy may not affect outcome of patients with glioblastoma: a secondary analysis from the radiation therapy oncology group database. J Clin Oncol 27:733-739, 2009

2. Burnet NG, Jena R, Jefferies SJ, Stenning SP, Kirkby NF: Mathematical modelling of survival of glioblastoma patients suggests a role for radiotherapy dose escalation and predicts poorer outcome after delay to start treatment. Clin Oncol (R Coll Radiol) 18:93-103, 2006 
3. Cancer Genome Atlas Research Network: Comprehensive genomic characterization defines human glioblastoma genes and core pathways. Nature 455:1061-1068, 2008

4. Do V, Gebski V, Barton MB: The effect of waiting for radiotherapy for grade III/IV gliomas. Radiother Oncol 57:131136,2000

5. Gorlia T, van den Bent MJ, Hegi ME, Mirimanoff RO, Weller M, Cairncross JG, et al: Nomograms for predicting survival of patients with newly diagnosed glioblastoma: prognostic factor analysis of EORTC and NCIC trial 26981-22981/CE.3. Lancet Oncol 9:29-38, 2008

6. Graus F, Bruna J, Pardo J, Escudero D, Vilas D, Barceló I, et al: Patterns of care and outcome for patients with glioblastoma diagnosed during 2008-2010 in Spain. Neuro Oncol 15:797-805, 2013

7. Irwin C, Hunn M, Purdie G, Hamilton D: Delay in radiotherapy shortens survival in patients with high grade glioma. J Neurooncol 85:339-343, 2007

8. Kirkby NF, Jefferies SJ, Jena R, Burnet NG: A mathematical model of the treatment and survival of patients with highgrade brain tumours. J Theor Biol 245:112-124, 2007

9. Lai R, Hershman DL, Doan T, Neugut AI: The timing of cranial radiation in elderly patients with newly diagnosed glioblastoma multiforme. Neuro Oncol 12:190-198, 2010

10. Lawrence YR, Blumenthal DT, Matceyevsky D, Kanner AA, Bokstein F, Corn BW: Delayed initiation of radiotherapy for glioblastoma: how important is it to push to the front (or the back) of the line? J Neurooncol 105:1-7, 2011

11. Noel G, Huchet A, Feuvret L, Maire JP, Verrelle P, Le Rhun E, et al: Waiting times before initiation of radiotherapy might not affect outcomes for patients with glioblastoma: a French retrospective analysis of patients treated in the era of concomitant temozolomide and radiotherapy. J Neurooncol 109:167-175, 2012

12. Noël G, Quetin P, Heymann S, Karamanoukian D, Schott R: [Diagnostic and treatment delays do not modify the treatment outcome of patients with multiform glioblastoma.] Cancer Radiother 13:17-23, 2009 (Fr)

13. Patel VN, Gokulrangan G, Chowdhury SA, Chen Y, Sloan
AE, Koyutürk M, et al: Network signatures of survival in glioblastoma multiforme. PLOS Comput Biol 9:e1003237, 2013

14. Stupp R, Hegi ME, Mason WP, van den Bent MJ, Taphoorn MJ, Janzer RC, et al: Effects of radiotherapy with concomitant and adjuvant temozolomide versus radiotherapy alone on survival in glioblastoma in a randomised phase III study: 5-year analysis of the EORTC-NCIC trial. Lancet Oncol 10:459-466, 2009

15. Stupp R, Mason WP, van den Bent MJ, Weller M, Fisher B, Taphoorn MJ, et al: Radiotherapy plus concomitant and adjuvant temozolomide for glioblastoma. N Engl J Med 352:987-996, 2005

16. Valduvieco I, Verger E, Bruna J, Caral L, Pujol T, Ribalta T, et al: Impact of radiotherapy delay on survival in glioblastoma. Clin Transl Oncol 15:278-282, 2013

17. Verhaak RG, Hoadley KA, Purdom E, Wang V, Qi Y, Wilkerson MD, et al: Integrated genomic analysis identifies clinically relevant subtypes of glioblastoma characterized by abnormalities in PDGFRA, IDH1, EGFR, and NF1. Cancer Cell 17:98-110, 2010

\section{Author Contributions}

Conception and design: Bloch, Sun, Ivan, Kaur, Parsa. Acquisition of data: Sun, Sayegh. Analysis and interpretation of data: Sun, Oh, Ivan, Clark, Safaee. Drafting the article: Sun. Critically revising the article: Bloch, Sun, Parsa. Reviewed submitted version of manuscript: Sun. Approved the final version of the manuscript on behalf of all authors: Bloch. Statistical analysis: Sun. Administrative/technical/material support: Oh, Safaee, Parsa. Study supervision: Ivan, Parsa.

\section{Correspondence}

Orin Bloch, Department of Neurological Surgery, Northwestern University, Feinberg School of Medicine, 676 N. St. Clair St., Ste. 2210, Chicago, IL 60611-2922. email: orin.bloch@ northwestern.edu. 